03

\title{
Структурные разновидности политипов
}

\author{
(C) Е.А. Беленков, В.А. Грешняков \\ Челябинский государственный университет, \\ Челябинск, Россия \\ E-mail: belenkov@csu.ru
}

(Поступила в Редакцию 20 марта 2017 г.)

Представлены результаты теоретического анализа структурных разновидностей соединений, для которых характерен политипизм. В результате установлена возможность существования изомерных разновидностей политипов, а также закономерности их формирования в зависимости от периода повторяемости слоев в упаковке. Кроме того, методами теории функционала плотности выполнены расчеты структуры и свойств различных политипных модификаций алмаза. Обнаружены зависимости межслоевых расстояний и разностной полной энергии от гексагональности политипов алмаза и их изомерных разновидностей. Рассчитаны теоретические порошковые рентгенограммы политипов алмаза и выполнен их сравнительный анализ.

Исследование выполнено при финансовой поддержке РФФИ в рамках научного проекта № 16-3300030 мол_а и Фонда перспективных научных исследований Челябинского государственного университета.

DOI: 10.21883/FTT.2017.10.44957.088

\section{1. Введение}

Структурные разновидности соединений, имеющих одинаковый химический состав, обладают различными свойствами. Диапазон изменения свойств зависит от того, какие это структурные разновидности - аллотропные, полиморфные или политипные.

Наиболее сильно свойства отличаются для аллотропных модификаций. Примером аллотропных разновидностей углеродных соединений являются карбин, графит и алмаз. Значительное отличие свойств этих соединений обусловлено различными гибридизациями электронных орбиталей углеродных атомов. В карбине гибридизация $s p^{1}$, в графите $-s p^{2}$, в алмазе - $s p^{3}$. Конфигурация электронных орбиталей внешней оболочки углеродного атома при различной гибридизации отличается значительно, поэтому сильно отличаются свойства аллотропных разновидностей.

Следующий класс структурных разновидностей соединений (полиморфные) характеризуется меньшим диапазоном изменения свойств. В структуре полиморфов основная гибридизация электронных орбиталей одинакова, однако она искажается за счет изменения положения соседних атомов в первой координационной сфере, в результате чего углы между ковалентными связями отличаются от значений, характерных для основных аллотропных модификаций. Поэтому для углеродной системы полиморфы могут быть на основе карбина (карбиноидные материалы), графита (графитоподобные материалы) или алмаза (алмазоподобные материалы), гибридизированные состояния электронных орбиталей у углеродных атомов в них могут рассматриваться как искаженные основные состояния: $s p^{1 \pm \delta}, s p^{2 \pm \delta}$ и $s p^{3 \pm \delta}$ соответственно. Менее значимые отличия электронной конфигурации атомов в полиморфах по сравнению с аллотропами обуславливает меньший диапазон варьирования свойств.

Третий класс структурных разновидностей - политипы. Это структурные разновидности, отличающиеся порядком упаковки молекулярных или атомных слоев. Электронные конфигурации атомов у этих соединений практически не отличаются друг от друга. Это связано с тем, что относительный порядок атомов в первой координационной сфере практически одинаков. Значимые отличия структуры наблюдаются только в дальних координационных сферах. Поэтому свойства политипов должны отличаться незначительно, хотя имеются сообщения о значительных отличиях механических свойств кубического алмаза (политип $3 C$ ) и лонсдейлита (политип 2H) [1]. Количество возможных политипных разновидностей, например, $\mathrm{SiC}$ превышает две сотни [2]. Политипизм характернен для различных классов веществ: металлов, керамик, минералов, интерметаллидов, органических веществ [3-12]. Причем он обнаружен при изучении не только монокристаллов, но и пленок, частиц порошков, поликристаллических компактных материалов $[3,13]$. Различные политипы получаются в результате различного порядка упаковки слоев. Основным отличием политипов друг от друга считается количество слоев, через которое периодически повторяется структура. Механизмы образования политипных фаз недостаточно изучены, и в большинстве случаев не удается предсказать заранее, какой политип получится при различных экспериментальных методах синтеза и, следовательно, объяснить причины образования различных политипов при одинаковых термодинамических условиях. Также остается неясным, какова возможная структура всех различных политипных разновидностей. Поэтому в настоящей работе был выполнен теоретический анализ закономерностей формирования структуры политипов и их изомерных форм. 


\section{2. Структура политипных модификаций конденсированных фаз}

Явление политипизма характерно для соединений, в структуре которых можно выделить слоевые структурные элементы. Типичные соединения, в которых наблюдаются политипы, можно разделить на две группы: первая - вещества с алмазоподобной кристаллической решеткой (C, Si, SiC, $\left.\mathrm{ZnS}, \mathrm{AlN}, \mathrm{BN}, \mathrm{B}_{4} \mathrm{C}, \ldots\right)$, в которой все связи преимущественно ковалентные; вторая - вещества с гексагональной слоевой решеткой типа графита $(\mathrm{C}, \mathrm{BN}, \ldots)$, где связи - ковалентные (в слоях) и вандер-ваальсовые (между слоями). Подобие этих групп соединений заключается в том, что их структуры можно модельно рассматривать как состоящие из плотноупакованных сфер равного диаметра. Переход от конкретной структуры соединения к модельному представлению происходит в результате выбора группы атомов, которая рассматривается как молекула и заменяется в структуре на сферу. Например, в алмазной структуре сферой заменяется группа из пары соседних углеродных атомов, связанных ковалентной связью, в бинарных соединениях $\mathrm{SiC}, \mathrm{ZnS}, \mathrm{AlN}$ происходит замена сферами пар соседних атомов разных элементов, а в графитоподобных структурах заменяемая группа включает шесть атомов одного или разных элементов.

Сферы упакованы в слои. Симметрия такого гексагонального плотноупакованного слоя описывается слоевой группой $p 6 m m$ (№ 77). Каждый слой можно рассматривать как слой молекул-сфер с плотной шаровой упаковкой, так что слои лежат в плоскости $X Y$ и упаковываются в трехмерную структуру, наслаиваясь друг на друга по оси $Z$. Особенность замен такова, что для первой группы соединений с алмазным типом решетки замена пар атомов на сферы происходит так, что межатомная связь направлена вдоль оси $Z$ (рис. $1, a)$, для второй группы соединений с графитоподобной решеткой сфера содержит шесть атомов, ковалентные связи между которыми ориентированы в плоскости $X Y$ (рис. $1, b)$.

Поэтому необходимо установить закономерности формирования структуры в модельном представлении, после чего последующий обратный переход от сфер-молекул к атомам позволит определить причины образования политипных модификаций конкретных соединений.

Рассмотрим трехмерную структуру политипных модификаций в модельном представлении. Если первый слой сфер-молекул обозначить как слой типа $A$, то следующие слои можно плотно наложить таким образом, что молекулы второго слоя расположатся в позициях $\boldsymbol{\Delta}$ либо $\boldsymbol{\nabla}$ (рис. 2), это слои $B$ и $C$ типов соответственно. Таким образом, соседние слои при плотной упаковке не могут быть одинакового типа, период повторяемости слоев может быть равен 2, 3, 4 и т.д. Политипы отличаются друг от друга, во-первых, порядком чередования и периодом повторяемости слоев в направлении,
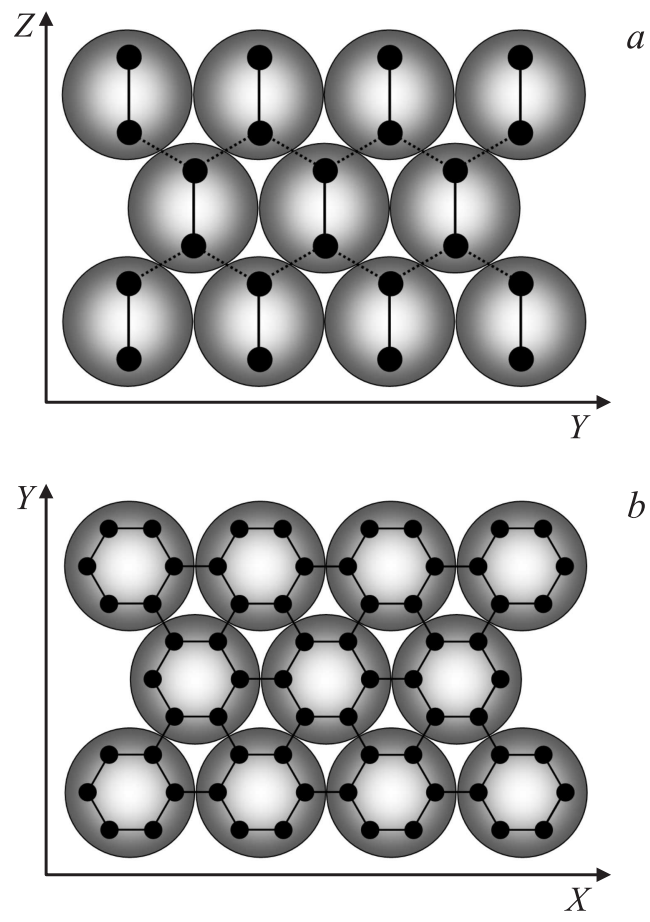

Рис. 1. Слой молекул с плотной шаровой упаковкой: (a) политипы с алмазоподобной кристаллической решеткой; (b) политипы с графитоподобной кристаллической решеткой.

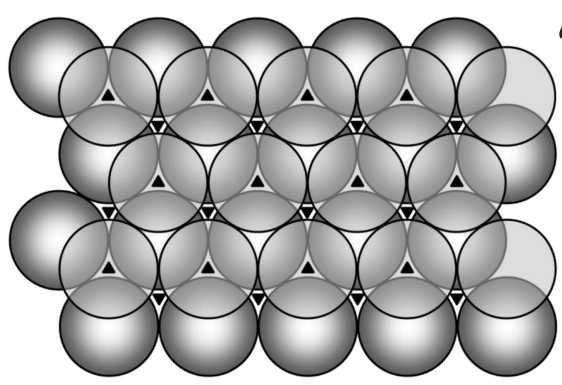

$a$

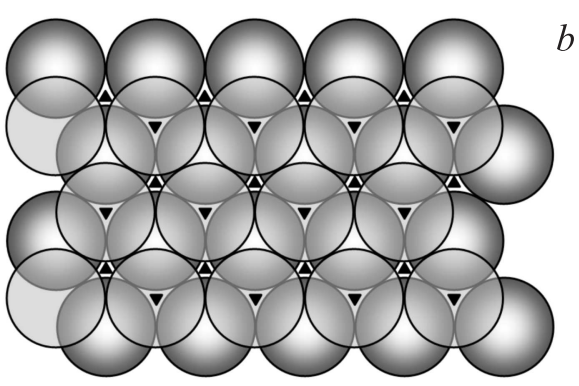

Рис. 2. Возможные варианты наложения слоев: $(a)$ позиция слоя $B$ относительно слоя $A ;(b)$ позиция слоя $C$ относительно слоя $A$.

перпендикулярном плоскости слоев. Во-вторых, отличия обусловлены типом примитивной элементарной ячейки. Например, период повторяемости слоев политипа $A B$ равен двум, а его примитивная элементарная ячейка относится к гексагональной сингонии $(H)$, обозначение 
политипа $2 H$. Аналогично, период повторяемости слоев политипа $A B C$ равен трем, примитивная элементарная ячейка относится к кубической сингонии $(C)$, обозначение этого политипа $3 C$.

В-третьих, политипы могут иметь различную гексагональность (Hex), численное значение которой определяют как долю гексагональных слоев, выраженную в процентах.

$$
\text { Hex }=\frac{k}{N} \cdot 100 \%,
$$

где $k-$ количество гексагональных слоев, $N-$ полное число слоев в элементарной ячейке политипа. Гексагональным считается слой, у которого соседние слои имеют одинаковые обозначения (например, в последовательности слоев $A B A$ слой $B$ является гексагональным, так как оба соседних слоя имеют обозначение $A$, в то время как в последовательности слоев $A B C$ слой $B$ не является гексагональным, поскольку соседние слои имеют разные обозначения $A$ и $C$ ). Рассмотрим, какие политипы могут образовываться при различных периодах повторяемости слоев.

\section{3. Методическая часть}

Анализ структуры возможных политипных модификаций выполняли путем перебора при помощи специально разработанной программы всех возможных комбинаций чередования слоев для политипных фаз, имеющих период повторяемости $N$ от 2 до 13. Так как в реальных кристаллах не все эти комбинации могут быть реализованы, то часть из них отбрасывали. К отбрасываемым были отнесены комбинации, в которых соседние слои относятся к одному типу, например, $A A, B B$ и т.д.; эквивалентные комбинации, т. е. соответствующие одной и той же кристаллической структуре при различном обозначении слоев. После отбрасывания таких комбинаций остаются только разрешенные. Далее из разрешенных комбинаций отбрасывали эквивалентные комбинации, которые получаются при заменах обозначений типов слоев. Всего возможно пять замен:

$$
\begin{array}{llll}
\text { 1. } & A \rightarrow B & B \rightarrow C & C \rightarrow A \\
\text { 2. } & A \rightarrow A & B \rightarrow C & C \rightarrow B \\
\text { 3. } & A \rightarrow C & B \rightarrow A & C \rightarrow B \\
\text { 4. } & A \rightarrow B & B \rightarrow A & C \rightarrow C \\
\text { 5. } & A \rightarrow C & B \rightarrow B & C \rightarrow A
\end{array}
$$

Кроме того, отбрасывались комбинации, которые можно представить как несколько повторов комбинаций с меньшим периодом повторяемости (например, структуpa $A B C A B C A B C$ с $N=9$ аналогична структуре $A B C$ с $N=3$ ), а также такие, которые переходят одна в другую при трансляционных сдвигах (например, структура $B C A$ аналогична структуре $A B C)$.
Геометрическая оптимизация структуры модельно построенных политипов и расчет их свойств были выполнены методом теории функционала плотности (DFT). Расчеты выполняли в программном пакете Quantum ESPRESSO [14] при использовании функционала обменно-корреляционной энергии Педью-БеркаЕрнзерхофа (РВЕ) [15]. Потенциалы ядер и остовных электронов атомов учитывались через сохраняющие норму псевдопотенциалы Труллера-Мартинса [16]. В кристалле блоховские электронные функции находились в ходе разложения по набору плоских волн, энергия отсечки которых составляла 60 Rydberg. Для всех расчетов была выбрана сетка $12 \times 12 \times 12$ в обратном пространстве.

Энергии сублимации $\left(E_{\mathrm{sub}}\right)$ политипов были найдены при помощи метода DFT-PBE как разность энергий изолированного атома углерода и атома, находящегося в связанном состоянии в кристалле. Также была вычислена разностная полная энергия $\left(\Delta E_{3 C}\right)$, представляющая собой разность полных энергий конкретного политипа и кубического алмаза в пересчете на один атом.

Расчет порошковых рентгенограмм выполнен по стандартной методике [17]. Рентгенограммы были рассчитаны для $\mathrm{Cu}-K_{\alpha}$ рентгеновского излучения.

\section{4. Результаты}

Структурные характеристики политипов, найденные в процессе перебора различных упаковок, представлены в табл. 1. Количество всех возможных комбинаций $(M)$ резко возрастает с увеличением периода повторяемости, согласно формуле $M=3^{N}$. После отбрасывания всех запрещенных комбинаций количество возможных политипов становится существенно меньше. Найдена зависимость количества возможных разрешенных комбинаций $\left(L_{N}\right)$ и разрешенных комбинаций за вычетом эквивалентных заменам $\left(P_{N}\right)$ от периода повторяемости слоев:

$$
\begin{aligned}
& L_{N}= \begin{cases}6, & \text { при } N=2 ; \\
2 L_{N-1}+6, & \text { при } N>2 \text { (четное); } \\
2 L_{N-1}-6, & \text { при } N>2 \text { (нечетное). }\end{cases} \\
& P_{N}= \begin{cases}1, & \text { при } N=2 ; \\
2 P_{N-1}+1, & \text { при } N>2 \text { (четное); } \\
2 P_{N-1}-1, & \text { при } N>2 \text { (нечетное). }\end{cases}
\end{aligned}
$$

Оставшееся количество структурных комбинаций $P_{N}$ все еще содержит долю тождественных структур, получающихся при трансляционных сдвигах. Количество возможных трансляционных сдвигов зависит от периода повторяемости и, к сожалению, записать формулу для расчета количества комбинаций за вычетом повторяющихся в результате трансляций не удается. На последнем этапе это количество комбинаций необходимо еще уменьшить за счет тех, которые можно представить как несколько 
Таблица 1. Структурные характеристики политипов $\left(N-\right.$ период повторяемости слоев в упаковке, $M_{N}-$ количество всех возможных комбинаций, $L_{N}-$ количество разрешенных комбинаций, $P_{N}-$ количество разрешенных комбинаций за вычетом эквивалентных, $F_{N}$ - количество уникальных политипов, Hex - гексагональность политипа, $W$ - вероятность формирования политипа, в скобках указаны комбинации, эквивалентные политипам с меньшими значениями $N$ )

\begin{tabular}{|c|c|c|c|c|c|c|c|}
\hline$N$ & $M_{N}$ & $L_{N}$ & $P_{N}$ & $F_{N}$ & Hex, \% & Чередование слоев & $W$ \\
\hline 2 & 9 & 6 & 1 & 1 & 100 & $A B A B \ldots$ & 100 \\
\hline 3 & 27 & 6 & 1 & 1 & 0 & $A B C A B C \ldots$ & 100 \\
\hline \multirow{2}{*}{4} & \multirow{2}{*}{81} & \multirow{2}{*}{18} & \multirow{2}{*}{3} & \multirow{2}{*}{1} & 50 & $A B A C \ldots$ & $66.67(100)$ \\
\hline & & & & & $(100)$ & $(A B A B \ldots)$ & $33.33(0)$ \\
\hline 5 & 243 & 30 & 5 & 1 & 40 & $A B A B C \ldots$ & 100 \\
\hline \multirow{4}{*}{6} & \multirow{4}{*}{729} & \multirow{4}{*}{66} & \multirow{4}{*}{11} & \multirow{4}{*}{2} & 66.7 & $A B A B A C \ldots$ & $54.55(66.67)$ \\
\hline & & & & & 33.3 & $A B A C B C \ldots$ & $27.27(33.33)$ \\
\hline & & & & & $(100)$ & $(A B A B \ldots)$ & $9.09(0)$ \\
\hline & & & & & $(0)$ & $(A B C A B C \ldots)$ & $9.09(0)$ \\
\hline \multirow{3}{*}{7} & \multirow{3}{*}{2187} & \multirow{3}{*}{126} & \multirow{3}{*}{21} & \multirow{3}{*}{3} & 57.1 & $A B A B A B C \ldots$ & 33.33 \\
\hline & & & & & 57.1 & $A B A B C A C \ldots$ & 33.33 \\
\hline & & & & & 28.6 & $A B A C A B C \ldots$ & 33.33 \\
\hline \multirow{8}{*}{8} & \multirow{8}{*}{6561} & \multirow{8}{*}{258} & \multirow{8}{*}{43} & \multirow{8}{*}{6} & 75 & $A B A B A B A C \ldots$ & $18.6(20)$ \\
\hline & & & & & 75 & $A B A B A C A C \ldots$ & $9.3(10)$ \\
\hline & & & & & 50 & $A B A B A C B C \ldots$ & $18.6(20)$ \\
\hline & & & & & 25 & $A B A B C A B C \ldots$ & $18.6(20)$ \\
\hline & & & & & 50 & $A B A B C B A C \ldots$ & $18.6(20)$ \\
\hline & & & & & 25 & $A B A C B A B C \ldots$ & $9.3(10)$ \\
\hline & & & & & $(100)$ & $(A B A B A B A B)$ & $2.33(0)$ \\
\hline & & & & & $(50)$ & $(A B A C A B A C)$ & $4.65(0)$ \\
\hline \multirow{11}{*}{9} & \multirow{11}{*}{19683} & \multirow{11}{*}{510} & \multirow{11}{*}{85} & \multirow{11}{*}{10} & 66.7 & $A B A B A B A B C \ldots$ & $10.59(10.71)$ \\
\hline & & & & & 66.7 & $A B A B A B C A C \ldots$ & $10.59(10.71)$ \\
\hline & & & & & 66.7 & $A B A B A B C B C \ldots$ & $10.59(10.71)$ \\
\hline & & & & & 44.4 & $A B A B A C A B C \ldots$ & $10.59(10.71)$ \\
\hline & & & & & 44.4 & $A B A B A C B A C \ldots$ & $10.59(10.71)$ \\
\hline & & & & & 44.4 & $A B A B C A B A C \ldots$ & $10.59(10.71)$ \\
\hline & & & & & 44.4 & $A B A B C A C B C \ldots$ & $10.59(10.71)$ \\
\hline & & & & & 44.4 & $A B A B C B A B C \ldots$ & $10.59(10.71)$ \\
\hline & & & & & 66.7 & $A B A B C B C A C \ldots$ & $3.53(3.57)$ \\
\hline & & & & & 22.2 & $A B A C B A C B C \ldots$ & $10.59(10.71)$ \\
\hline & & & & & $(0)$ & $(A B C A B C A B C \ldots)$ & $1.18(0)$ \\
\hline
\end{tabular}

повторов комбинаций с меньшим периодом повторяемости. В результате для каждого периода повторяемости получается число уникальных комбинаций $(F)$. Расчетные величины $F$ приведены в табл. 1. Таким образом, если период повторяемости слоев в политипе меньше шести, то существует по одному политипу для каждого определенного $N$. Начиная с шести слоев, одному и тому же числу $N$ может соответствовать несколько 
Таблица 1 (продолжение).

\begin{tabular}{|c|c|c|c|c|c|c|c|}
\hline $\mathrm{N}$ & $M_{N}$ & $L_{N}$ & $P_{N}$ & $F_{N}$ & Hex, \% & Чередование слоев & $W$ \\
\hline \multirow{20}{*}{10} & \multirow{20}{*}{59049} & \multirow{20}{*}{1026} & \multirow{20}{*}{171} & \multirow{20}{*}{18} & 80 & $A B A B A B A B A C \ldots$ & $5.85(6.06)$ \\
\hline & & & & & 80 & $A B A B A B A C A C \ldots$ & $5.85(6.06)$ \\
\hline & & & & & 60 & $A B A B A B A C B C \ldots$ & $5.85(6.06)$ \\
\hline & & & & & 40 & $A B A B A B C A B C \ldots$ & $5.85(6.06)$ \\
\hline & & & & & 60 & $A B A B A B C B A C \ldots$ & $5.85(6.06)$ \\
\hline & & & & & 60 & $A B A B A C A B A C \ldots$ & $5.85(6.06)$ \\
\hline & & & & & 60 & $A B A B A C A C B C \ldots$ & $5.85(6.06)$ \\
\hline & & & & & 40 & $A B A B A C B A B C \ldots$ & $5.85(6.06)$ \\
\hline & & & & & 60 & $A B A B A C B C A C \ldots$ & $5.85(6.06)$ \\
\hline & & & & & 60 & $A B A B A C B C B C \ldots$ & $2.92(3.03)$ \\
\hline & & & & & 40 & $A B A B C A B C A C \ldots$ & $5.85(6.06)$ \\
\hline & & & & & 40 & $A B A B C A B C B C \ldots$ & $5.85(6.06)$ \\
\hline & & & & & 40 & $A B A B C A C B A C \ldots$ & $5.85(6.06)$ \\
\hline & & & & & 60 & $A B A B C B A B A C \ldots$ & $2.92(3.03)$ \\
\hline & & & & & 40 & $A B A B C B A C B C \ldots$ & $5.85(6.06)$ \\
\hline & & & & & 40 & $A B A C A B A C B C \ldots$ & $5.85(6.06)$ \\
\hline & & & & & 20 & $A B A C A B C A B C \ldots$ & $5.85(6.06)$ \\
\hline & & & & & 20 & $A B A C B A C A B C \ldots$ & $2.92(3.03)$ \\
\hline & & & & & $(100)$ & $(A B A B A B A B A B \ldots)$ & $0.58(0)$ \\
\hline & & & & & $(40)$ & $(A B A B C A B A B C \ldots)$ & $2.92(0)$ \\
\hline \multirow{4}{*}{11} & \multirow{4}{*}{177147} & \multirow{4}{*}{2046} & \multirow{4}{*}{341} & \multirow{4}{*}{31} & 18.2 & 2 политипа & \multirow{4}{*}{3.23 для всех политипов } \\
\hline & & & & & 36.4 & 9 политипов & \\
\hline & & & & & 54.5 & 15 политипов & \\
\hline & & & & & 72.7 & 5 политипов & \\
\hline \multirow{14}{*}{12} & \multirow{14}{*}{531441} & \multirow{14}{*}{4098} & \multirow{14}{*}{683} & \multirow{14}{*}{60} & 16.7 & 1 политип & 1.76 \\
\hline & & & & & 16.7 & 1 политип & 0.88 \\
\hline & & & & & 33.3 & 14 политипов & 1.76 \\
\hline & & & & & 33.3 & 1 политип & 0.44 \\
\hline & & & & & 50.0 & 24 политипа & 1.76 \\
\hline & & & & & 50.0 & 3 политипа & 0.88 \\
\hline & & & & & 50.0 & 1 политип & 0.59 \\
\hline & & & & & 66.7 & 12 политипов & 1.76 \\
\hline & & & & & 83.3 & 2 политипа & 1.76 \\
\hline & & & & & 83.3 & 1 политип & 0.88 \\
\hline & & & & & $(100)$ & $(A B A B \ldots)$ & 0.15 \\
\hline & & & & & (0) & $(A B C A B C \ldots)$ & 0.15 \\
\hline & & & & & $(50)$ & $(A B A C A)$ & 0.29 \\
\hline & & & & & $(66.7)$ & $(A B A B A C)$ & 0.88 \\
\hline
\end{tabular}


Таблица 1 (продолжение).

\begin{tabular}{|c|c|c|c|c|c|c|c|}
\hline $\mathrm{N}$ & $M_{N}$ & $L_{N}$ & $P_{N}$ & $F_{N}$ & Hex, \% & Чередование слоев & $W$ \\
\hline \multirow{5}{*}{13} & \multirow{5}{*}{1594323} & \multirow{5}{*}{8190} & \multirow{5}{*}{1365} & \multirow{5}{*}{105} & 15.4 & 2 политипа & \multirow{5}{*}{0.95 для всех политипов } \\
\hline & & & & & 30.8 & 19 политипов & \\
\hline & & & & & 46.2 & 42 политипа & \\
\hline & & & & & 61.5 & 35 политипов & \\
\hline & & & & & 76.9 & 7 политипов & \\
\hline
\end{tabular}

уникальных политипов. Возможные чередования слоев представлены в табл. 1. Эти политипы отличаются не только порядком чередования слоев, но и такой характеристикой, как гексагональность. В результате анализа структуры политипов (табл. 1) была найдена закономерность:

$$
\text { Hex }=\frac{2 i}{N} \cdot 100 \%
$$

где

$$
i= \begin{cases}1 & \text { при } N=2 ; \\ 0 & \text { при } N=3 ; \\ 1, \ldots, i_{\max } & \text { при } N>3 ;\end{cases}
$$

и

$$
i_{\max }= \begin{cases}\frac{N}{2}-1, & N \text { четное; } \\ \frac{N-1}{2}-1, & N \text { нечетное }\end{cases}
$$

По этой формуле можно рассчитать гексагональности политипов с любыми периодами повторяемости $N$, а не только в пределах рассмотренных политипов с $N<14$. Для политипов с $N>5$ гексагональность принимает различные значения, и появляется несколько различных изомерных разновидностей для каждого политипа с $N=$ const.

Возникает вопрос, могут ли изомерные разновидности политипов быть обнаружены экспериментально и насколько сильно они отличаются по свойствам. Для решения этой задачи были выполнены расчеты оптимизированной структуры, энергетических параметров, электронных свойств и рентгенограмм.

Анализ геометрически оптимизированных структур политипных модификаций показал, что их кристаллические решетки могут относиться к следующим пространственным группам: $F d \overline{3} m, P 6_{3} / m m c, P \overline{6} m 2, R \overline{3} m$ и $P 3 m 1$. Элементарные ячейки практически всех политипов принадлежат к гексагональной и тригональной сингониям. Кубическая сингония соответствует только политипу $3 C$, т. е. кубическому алмазу.

Найденные в результате DFT-расчетов длины межатомных связей в различных политипах отличаются. Сравнительный анализ показал, что средние расстояния $d$ между соседними молекулярными слоями зависят от гексагональности политипа (рис. 3,a). Эта зависимость имеет линейный характер: $d=a+b H e x$, где $a=(2.076 \pm 0.003) \AA$ и $b=(249 \pm 5) \cdot 10^{-6} \AA$. Ход теоретически полученной зависимости хорошо соответствует экспериментальной зависимости для политипов $\mathrm{SiC}$, которую можно построить по данным работы [3]. Также прослеживается взаимосвязь между разностной полной энергией $\left(\Delta E_{3 C}\right)$ и гексагональностью политипов, однако зависимость имеет нелинейный характер (рис. $3, b$ ).

Также были рассчитаны такие свойства политипных разновидностей алмаза, как плотность $(\rho)$, ширина прямой $\left(\Delta_{\Gamma}\right)$ и минимальной непрямой $\left(\Delta_{\mathrm{DOS}}\right)$ запрещенных зон (табл. 2). Последующий анализ данных табл. 2 не

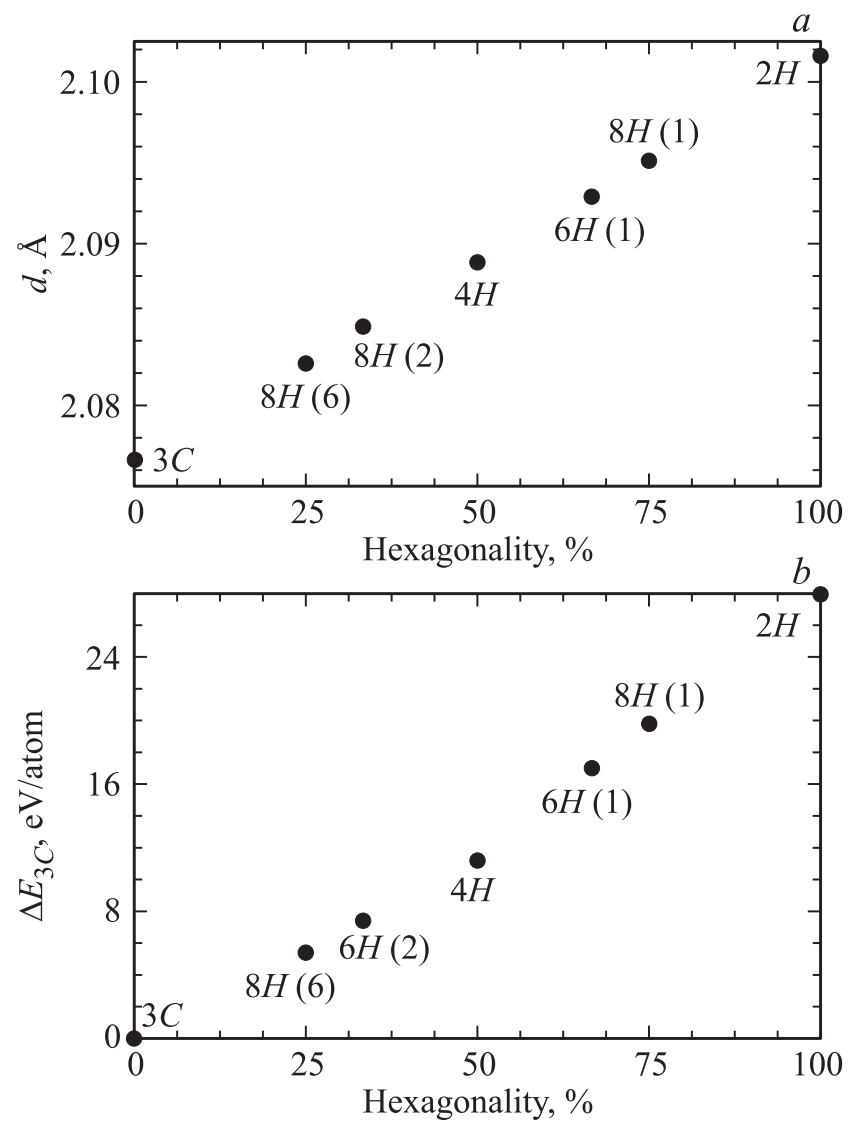

Рис. 3. Зависимости межслоевых расстояний $(a)$ и разностной полной энергии $(b)$ от гексагональности политипов алмаза. 
Таблица 2. Структурные параметры и свойства политипов алмаза

\begin{tabular}{c|c|c|c|c|c|c|c|c}
\hline Политип & Hех, \% & Пространственная группа & $a, \AA$ & $c, \AA$ & $\rho, \mathrm{g} / \mathrm{cm}^{3}$ & $E_{\text {sub }}, \mathrm{eV} / \mathrm{atom}$ & $\Delta_{\Gamma}, \mathrm{eV}$ & $\Delta_{\text {Dos, }} \mathrm{eV}$ \\
\hline $3 C$ & 0 & $F d \overline{3} m$ & 3.597 & 3.597 & 3.429 & 7.887 & 5.92 & 5.56 \\
$2 H$ & 100 & $P 6_{3} / m m c$ & 2.528 & 4.203 & 3.429 & 7.859 & 5.89 & 5.20 \\
$4 H$ & 50 & $P 6_{3} / m m c$ & 2.535 & 8.355 & 3.431 & 7.876 & 5.90 & 5.92 \\
$6 H(1)$ & 66.7 & $P \overline{6} m 2$ & 2.538 & 12.509 & 3.430 & 7.879 & 5.92 & 5.74 \\
$6 H(2)$ & 33.3 & $P 6_{3} / m m c$ & 2.533 & 12.557 & 3.431 & 7.870 & 5.91 & 5.88 \\
$8 H(6)$ & 25 & $P 6_{3} / m m c$ & 2.539 & 16.661 & 3.430 & 7.881 & 5.92 & 5.68 \\
$8 H(1)$ & 75 & $P 6_{3} / m m c$ & 2.532 & 16.761 & 3.430 & 7.867 & 5.90 & 5.70
\end{tabular}

показал четких взаимосвязей между структурными характеристиками и свойствами. Плотности и ширина прямых запрещенных зон различных политипов отличаются незначительно в пределах ошибок расчета. Заметные
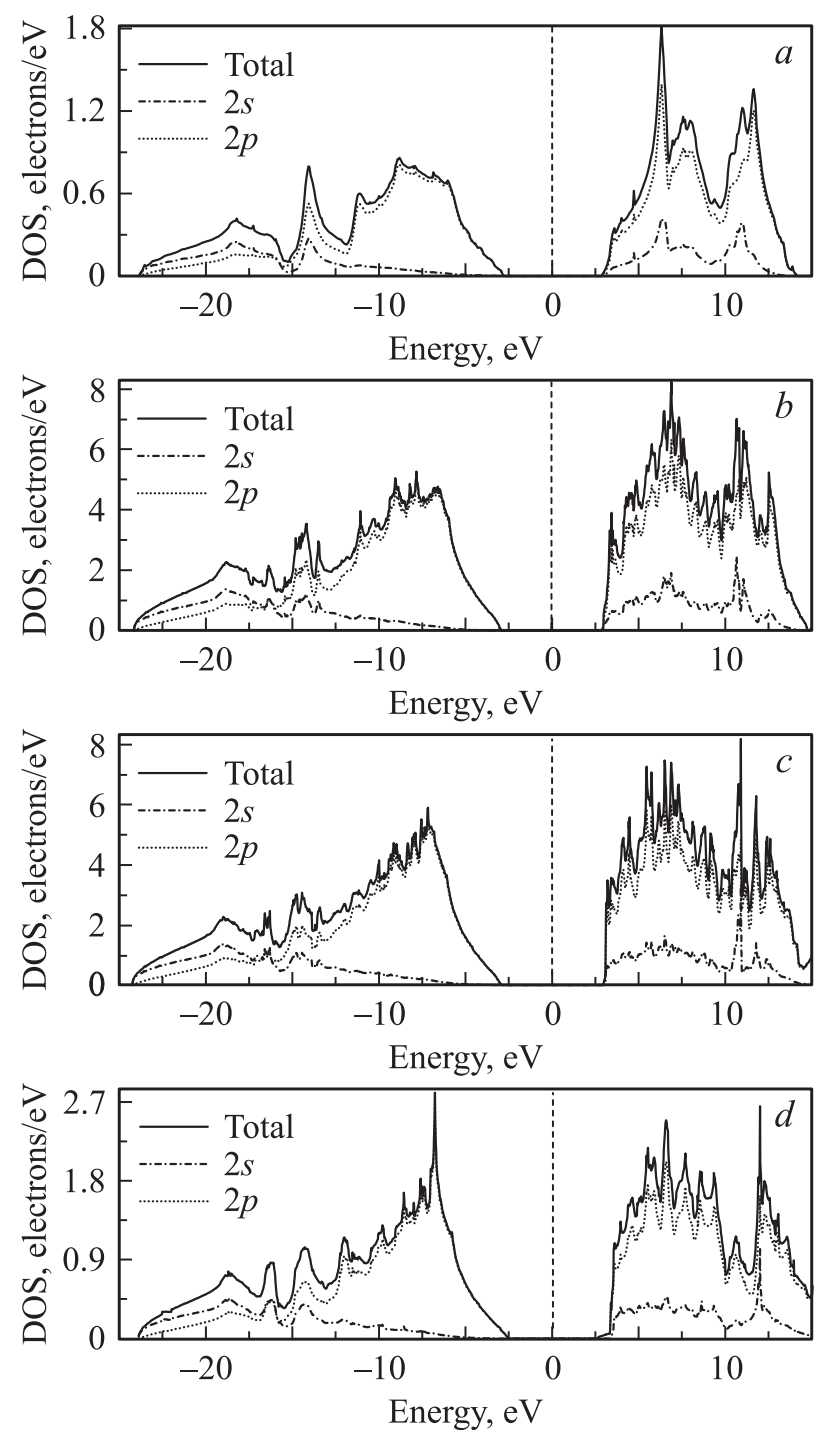

Рис. 4. Плотности электронных состояний политипов (энергия Ферми отмечена пунктирной линией и принята за начало отсчета): (a) 3C, Hex $=0 \%$; (b) $6 H$ (2), Hex $=33.3 \%$; (c) $6 \mathrm{H}(1)$, Hex $=66.7 \%$; (d) $2 \mathrm{H}$, Hex $=100 \%$.

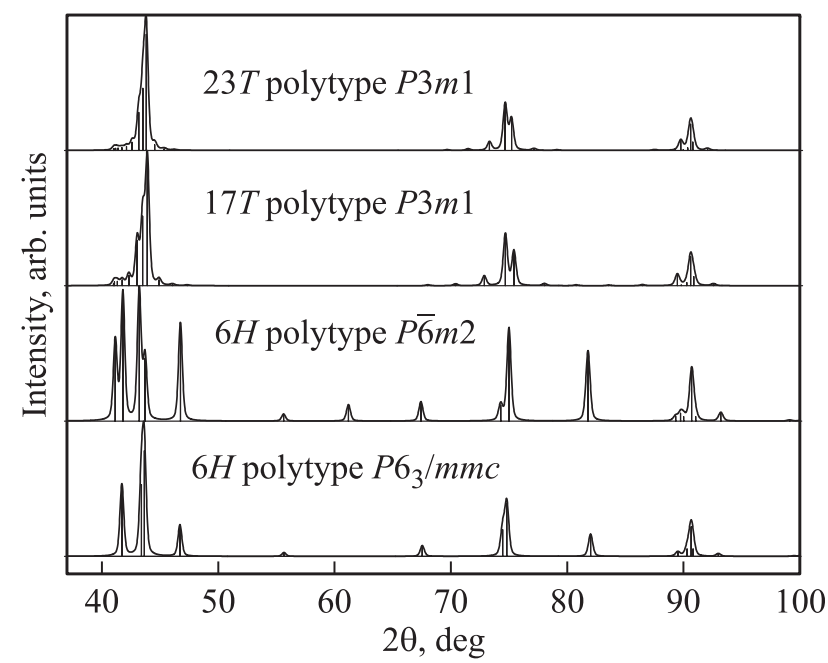

Рис. 5. Порошковые рентгенограммы различных политипов алмаза.

отличия наблюдаются только в картинах плотностей электронных состояний для политипов с достаточно большой разницей в их гексагональностях (рис. 4). Однако при этом ширина минимальной непрямой запрещенной зоны политипов отличается не более чем на $11 \%$.

Свойства различных политипов отличаются незначительно, однако, как показали теоретические расчеты их рентгенограмм, идентифицировать политипы возможно. Расчеты были выполнены для политипов с периодом повторяемости слоев от 2 до 30 и гексагональностью от 0 до 100\%. Наиболее интенсивные дифракционные максимумы политипов находятся в интервалах углов $2 \theta$ $41-48^{\circ}, 74-75^{\circ}$ и $90-91^{\circ}$. При увеличении периода повторяемости слоев $N$ и гексагональности значительно возрастает количество дифракционных максимумов на рентгенограмме, которые в результате сливаются в несколько „размытых“ максимумов (рис. 5). В результате сравнительного анализа рентгенограмм установлено, что для большинства политипных разновидностей с близкими значениями гексагональности нет значительных отличий в рентгенограммах (например, для политипов $17 T$ и $23 T$ на рис. 5), поэтому различать их сложно. Кроме того, иногда рентгенограммы изомерных политипных разновидностей отличаются сильнее, чем 
ренгенограммы политипов с разными периодами повторяемости слоев (рис. 5). Однозначно идентифицировать политип, вероятно, можно, если удастся синтезировать образцы, состоящие только из одного политипа. Если же образец представляет собой смесь политипов, то идентификация политипного состава становится практически невозможной. Аналогичные сложности возникают при идентификации политипов по электронограммам. Неопределенность при их анализе обусловлена тем, что дифракционным максимумам могут быть присвоены неправильные кристаллографические индексы для политипов с кратными периодами повторямости слоев. Так, например, рефлексы 100, 101, 112 и 202 для политипа $2 H$ могут соответствовать рефлексам 100, 102, 114 и 204 для политипа $4 H$ и т.д.

\section{5. Заключение}

В результате исследования установлена возможность существования изомерных разновидностей политипов для соединений, в которых наблюдается политипизм. Политипы с периодом повторяемости $N>5$ имеют ряд изомерных разновидностей с одинаковым $N$, но различной гексагональностью. В результате близкую или одинаковую гексагональность имеют сразу несколько политипов с различным периодом повторяемости; повидимому, они могут одновременно формироваться и устойчиво существовать при одинаковых значениях термодинамических параметров, так как их энергии сублимации принимают близкие значения. Изомерные структуры при одинаковом периоде $N$ могут иметь разную гексагональность. В результате анализа установлены закономерности, позволяющие предсказать количество изомеров для полипов с различным периодом $N$, а также их гексагональность. Кроме того, были рассчитаны оптимизированные структуры политипов алмаза, значения их структурных параметров, полные разностные энергии и энергии сублимации, плотности электронных состояний и ширина запрещенных зон, картины рентгеновской дифракции. Установлены зависимости межслоевых расстояний и разностной полной энергии от гексагональности политипов алмаза и их изомерных разновидностей.

Близкие значения физических характеристик политипов и их энергий сублимации, по-видимому, указывают на то, что в процессе формирования структуры при одинаковых термодинамических условиях могут одновременно формироваться различные политипы алмаза. Кроме того, в пределах одного кристалла может образовываться случайный порядок упаковки слоев. Поэтому кристаллы со структурой конкретного политипа, очевидно, нельзя получить. Случайный порядок упаковки, возникновение или исчезновение дефектов упаковки может происходить при движении дислокаций в кристалле. В предельном случае за счет множества таких сдвигов могут происходить фазовые превращения одних политипов в другие (например, $2 H$ в $3 C$ и наоборот). Экспериментально доказать существование конкретных политипов и их изомерных форм по данным дифракционного анализа затруднительно из-за большого количества самых интенсивных дифракционных максимумов, близких по угловым положениям. Эти максимумы могут быть значительно „размыты“ для политипов с большими значениями периода повторяемости слов или его случайной упаковкой слоев. К настоящему времени экспериментально получены различные политипы алмаза: $2 H[18], 3 C, 4 H[19], 6 H[20,21], 8 H[22,23]$, $9 R[24], 15 R[25], 21 R$ [26] и др. Однако выводы об их наличии были сделаны по дифракционным данным электронографии и рентгеноструктурного анализа, которые, как указано выше, не могут быть строгим доказательством. Например, широко распространенное мнение о том, что импактные наноалмазы, сформировавшиеся в метеоритных кратерах, имеют структуру политипа $2 H$ (лонсдейлита), вероятно, является ошибочным. Эти наноалмазы, по-видимому, имеют случайный порядок упаковки слоев [27].

\section{Список литературы}

[1] Z. Pan, H. Sun, Yi Zhang, C. Chen. Phys. Rev. Lett. 102, 5, 055503 (2009).

[2] А.С. Тарабанов, В.И. Костиков. Силицированный графит. Металлургия, М. (1977). 208 с.

[3] Г.С. Олейник, Н.В. Даниленко. Успехи химии 66, 615 (1997).

[4] А. Верма, П. Кришна. Полиморфизм и политипизм в кристаллах. Мир, М. (1969). 276 с.

[5] Г.Г. Гнесин. Карбидкремниевые материалы. Металлургия, M. (1977). 2016 c

[6] A. Machida, A. Ohmura, T. Watanuki, K. Aoki, K. Takemura. Phys. Rev. B 76, 052101 (2007).

[7] M. Durandurdu. Phys. Rev. B 75, 235204 (2007).

[8] H. Kuwamoto. J. Mater. Sci. Lett. 4, 8, 940 (1985).

[9] G.D. Price. Phys. Chem. Minerals 10, 2, 77 (1983).

[10] J. Ingrin. Phys. Chem. Minerals 20, 1, 56 (1993).

[11] D.G. Howitt. J. Mater. Sci. 16, 5, 1249 (1981).

[12] W.J. Yu, M. Lau, S.P. Chan, Z.F. Liu, Q.Q. Zheng. Phys. Rev. B 67, 014108 (2003).

[13] А.В. Семенов, А.В. Лопин. Поверхность, рентгеновские, синхротронные и нейтронные исследования 9, 99 (2004).

[14] P. Giannozzi, S. Baroni, N. Bonini, M. Calandra, R. Car, C. Cavazzoni, D. Ceresoli, G.L. Chiarotti, M. Cococcioni, I. Dabo, A.D. Corso, S. de Gironcoli, S. Fabris, G. Fratesi, R. Gebauer, U. Gerstmann, C. Gougoussis, A. Kokalj, M. Lazzeri, L. Martin-Samos, N. Marzari, F. Mauri, R. Mazzarello, S. Paolini, A. Pasquarello, L. Paulatto, C. Sbraccial, S. Scandolo, G. Sclauzero, A.P. Seitsonen, A. Smogunov, P. Umari, R.M. Wentzcovitch. J. Phys.: Condens. Matter. 21, 39, 395502 (2009).

[15] J.P. Perdew, K. Burke, M. Ernzerhof. Phys. Rev. Lett. 77, 18, 3865 (1996).

[16] N. Troullier, J.L. Martins. Phys. Rev. B 43, 3, 1993 (1991).

[17] Я.С. Уманский, Ю.А. Скаков, А.Н. Иванов, Л.Н. Расторгуев. Кристаллография, рентгенография и электронная микроскопия. Металлургия, М. (1982). 632 с. 
[18] F.P. Bundy, J.S. Kasper. J. Chem. Phys. 46, 9, 3437 (1967).

[19] A.K. Sharma, H.G. Salunke, G.P. Das, P. Ayyub, M.S. Multani. J. Phys.: Condens. Matter. 8, 31, 5801 (1996).

[20] M. Frenklach, R. Kematick1, D. Huang, W. Howard, K.E. Spear, A.W. Phelps, R. Koba. J. Appl. Phys. 66, 1, 395 (1989).

[21] S. Bhargava, H.D. Bist, S. Sahli, M. Aslam, H.B. Tripathi. Appl. Phys. Lett. 67, 12, 1706 (1995).

[22] R. Kapil, B.R. Mehta, V.D. Vankar. Thin Solid Films 312, 1, 106 (1998).

[23] Z. Wang, Y. Zhao, C.-S. Zha, Q. Xue, R.T. Downs, R.-G. Duan, R. Caracas, X. Liao. Adv. Mater. 20, 3303 (2008).

[24] Y. Lifshitz, X.F. Duan, N.G. Shang, Q. Li, L. Wan, I. Bello, S.T. Lee. Nature 412, 404 (2001).

[25] R. Kapil, B.R. Mehta, V.D. Vankar. Appl. Phys. Lett. 68, 18, 2520 (1996).

[26] Y.G. Gogotsi, A. Kailer, K.G. Nickel. J. Appl. Phys. 84, 3, 1299 (1998).

[27] В.А. Грешняков, Е.А. Беленков. ЖЭТФ 151, 2, 310 (2017). 